\title{
Effet de la mobilité du soufre et ses dérivés sur la qualité des eaux dans un contexte de dépôt mal contrôlé : étude de cas
}

\author{
Daouda MAMA ${ }^{1^{*}}$, Martin AINA ${ }^{2}$, Benjamin $\mathrm{YAO}^{3}$, Véronique DELUCHAT ${ }^{1}$, \\ Moussa BOUKARI ${ }^{2}$, Bonaventure GUEDEGBE ${ }^{4}$ et Michel BAUDU $^{1}$ \\ ${ }^{1}$ Laboratoire Groupe de Recherche Eau Sol Environnement (GRESE), Faculté des Sciences, 123, Avenue \\ Albert Thomas, F-87060 Limoges Cedex, France. \\ ${ }^{2}$ Laboratoire d'Hydrologie Appliquée de la Faculté des Sciences et Techniques, 01 BP 526 Cotonou (Bénin) \\ ${ }^{3}$ Laboratoire de Procédés Industriels de Synthèse et de Environnement INP - HB \\ BP 1093 Yamoussoukro (Côte d'Ivoire): \\ ${ }^{4}$ Agence Béninoise pour l'Environnement (ABE),03 BP 4327 Cotonou (Bénin). \\ "Corresponding author; E-mail: mkdaouda@yahoo.fr ; Tél. (229) $90925128 / 9663$ 81 24;
}

\section{RESUME}

Le soufre solide au contact de l'eau à température élevée, peut par oxydation produire des dérivées qui peuvent contribuer directement ou indirectement à la dégradation des matériaux et de la qualité des milieux récepteurs (air, sol et eau) et avoir des effets nocifs sur la santé. A Parakou, au nord du Bénin (Afrique de l'Ouest), se pratique depuis plus de 10 ans, le stockage à l'air libre du soufre granulaire (16 000 tonnes en moyenne par an), qui est une marchandise en transit pour la République du Niger. L'objectif du présent article est de mettre en relief la mobilité du soufre de ce dépôt et son impact sur la qualité de la ressource en eau. Les acides dérivés du soufre et les métaux toxiques issus de la corrosion des matériaux autour du site, ont eu pour conséquence la dégradation de la qualité des eaux des puits par la modification des caractéristiques physico-chimiques et microbiologiques avec :- un taux moyen variant de 282 clostridium perfergens / $100 \mathrm{ml}$ d'eau (puits hors de la zone de drainage) et 13018 clostridium perfergens / $100 \mathrm{ml}$ (puits situés en zone de drainage du soufre) ; - des concentrations de $0,01 \mathrm{mg} \mathrm{Pb} / \mathrm{L}$ et $0,05 \mathrm{mg} \mathrm{Zn/L} \mathrm{(puits} \mathrm{hors} \mathrm{site} \mathrm{de} \mathrm{stockage)} \mathrm{à}$ $1,55 \mathrm{mg} \mathrm{Pb} / \mathrm{L}$ et $0,39 \mathrm{mg} \mathrm{Zn} / \mathrm{L}$ (puits en zone de drainage). Le rôle joué par les eaux de ruissellement, le vent et les fortes températures (climat subtropical) a été pris en compte et mis en relief.

(C) 2011 International Formulae Group. All rights reserved.

Mots clés : Dépôt de soufre, Mobilité, Impacts, Qualité, Eau, Parakou.

\section{INTRODUCTION}

Les ions sulfates $\left(\mathrm{SO}_{4}{ }^{2-}\right)$ dans les eaux de l'aquifère éocène des Sables inframolassiques permettent de distinguer deux sources: une première liée à la dissolution du gypse $\left(\mathrm{CaSO}_{4}, \mathrm{H}_{2} \mathrm{O}\right)$, présent au toit de l'aquifère, et la seconde liée à l'oxydation de la pyrite (André et al., 2002). Les propriétés géochimiques induisent des modifications de composition chimique. Les zones de bioréduction du soufre peuvent exister dans les différents bassins hydrogéologiques (André, 2002).

Les particules en suspension contenant du soufre $\left(\mathrm{SO}_{\mathrm{x}}\right)$ et du dioxyde de soufre peuvent être facilement inhalées, puis pénétrer 
dans les poumons, où elles sont à l'origine de maladies des bronches. L'acide sulfurique, hydroscopique, est très irritant pour les voies respiratoires supérieures (Le Roux et al., 1997 ; Wind et al., 1995,Lawe et al., 1993 ; Sigg et al., 1994). Les effets à court terme d'une exposition à de fortes concentrations en $\mathrm{SO}_{2}$ dans 1'air, conduisent à l'accroissement soudain du nombre de décès, des admissions à l'hôpital et des affaiblissements temporaires des fonctions pulmonaires. Les effets à long terme concernent la fréquence accrue des infections respiratoires (enfants), la prévalence accrue des symptômes respiratoires (adultes et enfants), des taux de bronchites plus élevés dans les zones polluées comme l'indiquent les travaux de: Braun et al. (1992), Chailleux et al. (1990); Dab et al. (1995) et Lameloise (1996).

Le soufre réagit avec la plupart des métaux et conduit à la formation de précipités MS avec les cations divalents. L'émission du dioxyde de soufre ou sa production dans l'air par oxydation sont à l'origine des pluies acides et ont des conséquences sur la qualité des plans d'eau, des nappes phréatiques, des forêts et des sols. Les effets de l'acidification varient géographiquement et dépendent d'une combinaison de deux facteurs : la quantité de dépôts (sec et humide) et la sensibilité naturelle du milieu récepteur (sol et eau). Dans le cas de fortes teneurs en $\mathrm{SO}_{\mathrm{x}}$, les pluies acides conduisent à la dégradation des matériaux, des toitures et des objets du patrimoine culturel. En effet, la chute du pH entraîne une solubilisation importante des métaux comme le fer, le zinc, le chrome, le cuivre et le plomb (Benmoussa et al., 1994). Ainsi, ces métaux vont être libérés et se trouver sous forme mobile $\left(\mathrm{M}^{\mathrm{n}+}\right)$ dans les eaux et les sols.

Le Niger, pour l'exploitation de son gisement d'uranium, a besoin d'acide sulfurique pour produire des composés d'étape $\left(\mathrm{UO}_{2} \mathrm{SO}_{4}\right)$. Le soufre granulaire importé à cet effet, transite par la gare ferroviaire de la ville de Parakou où il est stocké en attendant son transfert au Niger par camions. Ainsi, ce produit entreposé à ciel ouvert et dont une bonne partie est drainée par les eaux de pluie et transportée par le vent, a des conséquences sur l'environnement et les ressources en eau. Le soufre solide stocké en vrac dégage naturellement un peu d'hydrogène sulfuré à partir de la teneur initiale (200 à 300 ppm), variable selon l'origine et le mode de fabrication, mais ce dégazage est très lent (Le Cedre, 2010).

Une observation de l'environnement immédiat de l'entrepôt de soufre permet de constater que des habitations sont abandonnées et que la majorité des toitures en tôles sont rouillées et parfois perforées. La végétation autour des habitations est peu développée, l'herbe ne pousse pas à certains endroits même en l'absence d'entretien. En effet, les sulfures produits par les bactéries anaérobies utilisent les formes oxydées du soufre (sulfates et thiosulfate) et inhibent la germination des plantes (Freney et al., 1982). Un déversement ne peut entraîner que très exceptionnellement la présence de soufre colloïdal en suspension et nuire alors à la biocénose aquatique (effets observés à des teneurs comprises entre 1,6 et $10 \mathrm{~g} / \mathrm{l})$. On connaît sur les sols le pouvoir biocide du soufre vis-à-vis des parasites des plantes (Cedre, 2010).

Selon une étude de la société SERHAU-SEM (1999), le réseau d'alimentation en eau de la ville subit une forte dégradation due à la corrosion des conduites.

Une relation très significative est retrouvée à Saint-Nazaire (France) entre les pics de $\mathrm{SO}_{2}$ et les consultations pour crise d'asthme chez les enfants (Chailleux et al., 1990. Marzin et al., 1993). Ces travaux ont permis d'observer qu'un nombre plus élevé de crise d'asthme lors des fortes teneurs en $\mathrm{SO}_{2}$, $\mathrm{NO}_{2}, \mathrm{SO}_{4}$ avec les pressions atmosphériques élevées, mais seul le $\mathrm{SO}_{2}$ et la pression atmosphérique sont significativement corrélés avec le nombre journalier de crises. Soyseth et al. (1995) montrent que l'exposition des enfants dès les trois premières années de vie au dioxyde de soufre présent dans 
l'atmosphère, augmente significativement le risque d'apparition d'une hyperbronchie.

L'étude épidémiologique menée par Mama et al. (2001), a révélé des taux importants de pneumopathies (26\%) dans le quartier proche du dépôt contre $11 \%$ pour le quartier témoin; une forte fréquence des antécédents d'asthme (18\%) dans le quartier proche du dépôt contre une fréquence de $3 \%$ pour le quartier témoin Donkparou.

Selon les résultats des travaux de caractérisation des sols menés par Mama et al. (2001), il y a eu des apports réguliers de soufre et ses dérivés dans ces sols à partir du dépôt. Cet apport évolutif par sédimentation, a conduit à l'acidification des sols ( $\mathrm{pH}$ compris entre 1,6 et 5,9 pour le premier horizon $<20$ $\mathrm{cm}$ ), et présente déjà des possibilités de contamination des nappes.

L'étude de la contamination des ressources en eau envisagée ici pose le problème des effets néfastes qu'une mauvaise gestion des produits chimiques comme le soufre peuvent engendrer.

Le soufre granulé, solide cristallisé jaune citron, cassant, dit soufre $\alpha$, exposé à l'air libre et soumis aux intempéries, se solubilise et est transporté dans les eaux et les sols. Il se produit de multiples réactions d'hydrolyse ou d'oxydation. Ainsi on peut avoir les réactions suivantes:

- Dans l'air on aura une oxydation du soufre pour donner du dioxyde de soufre qui peut à son tour s'oxyder pour donner du trioxyde de soufre suivant l'équation :

$$
\mathrm{SO}_{2}+1 / 2 \mathrm{O}_{2} \rightarrow \mathrm{SO}_{3}
$$

- En présence de l'humidité, le trioxyde de soufre donnera de l'acide sulfurique suivant l'équation: $\mathrm{SO}_{3}+\mathrm{H}_{2} \mathrm{O} \rightarrow \mathrm{H}_{2} \mathrm{SO}_{4}$.

- Dans l'eau, on a: $3 \mathrm{~S}+2 \mathrm{H}_{2} \mathrm{O} \rightarrow 2 \mathrm{H}_{2} \mathrm{~S}+$ $\mathrm{SO}_{2}$. Le soufre se dissous dans les solutions aqueuses de sulfure alcalin pour donner des polysulfures. (Heslop et Robinson, 1973).

Les réactions d'oxydo-réduction aboutissent à la prépondérance de la forme oxydée ou réduite selon les conditions du milieu et conduisent entre autres à la formation des acides sulfureux $\left(\mathrm{H}_{2} \mathrm{SO}_{3}\right)$ et sulfurique $\left(\mathrm{H}_{2} \mathrm{SO}_{4}\right)$, des sulfates $\left(\mathrm{SO}_{4}{ }^{2-}\right)$, des thiosulfates $\left(\mathrm{S}_{2} \mathrm{O}_{3}{ }^{2-}\right)$, des trithionates $\left(\mathrm{S}_{3} \mathrm{O}_{6}{ }^{2-}\right)$, des dithionates $\left(\mathrm{S}_{2} \mathrm{O}_{6}{ }^{2-}\right)$, des pentathionates $\left(\mathrm{S}_{5} \mathrm{O}_{6}{ }^{2-}\right)$ de l'hydrogène sulfuré $\left(\mathrm{H}_{2} \mathrm{~S}\right)$ (Lawe et al., 1993 ; Sigg et al., 1994). L'objectif de ce travail est de montrer que le dépôt constitue la principale source de pollution et de contamination en soufre et est à l'origine de la dégradation de la qualité des ressources en eau de la ville de Parakou.

\section{MATERIEL ET METHODES}

Site

Le dépôt de soufre est situé à la gare ferroviaire de la ville de Parakou (qui se trouve à $9^{\circ} 20^{\prime}$ de latitude Nord et à $2^{\circ} 35^{\prime}$ de longitude Est; avec une altitude moyenne de 370 m), au Bénin en Afrique de l'Ouest. Ce dépôt, à ciel ouvert, se trouve dans le quartier dénommé «dépôt» (en référence à l'entrepôt).

Les Figures 1 et 2 illustrent la position du site de dépotage du soufre et les conditions de ce dépotage à la gare ferroviaire de Parakou. Le vent et la pluie contribuent au transport du soufre hors du site de dépôt.

\section{Choix des points d'échantillonnage}

Le dépôt de soufre a été pris comme un point de repère et noté $A_{0}$. 25 points de prélèvement notés de $\mathrm{A}_{1}$ à $\mathrm{A}_{25}$ ont été retenus en tenant compte de la disponibilité des puits, $\mathrm{du}$ sens de drainage par ruissellement du soufre. A cet effet, le côté Est a été privilégié avec le suivi du principal cours d'eau de la ville qui reçoit ces éléments de drainage à savoir 'Boundarou'.

Un prélèvement d'eau de pluie stagnante a été effectué au point $\mathrm{A}_{24}$ situé à environ $50 \mathrm{~m}$ du lieu de stockage. La hauteur d'eau stagnante est de l'ordre $15 \mathrm{~cm}$, l'oxygénation de l'eau est assurée par les échanges air/eau. Le prélèvement d'eau potable de la ville (échantillon témoin), a été fait au point $\mathrm{A}_{25}$ au robinet d'un abonné. La présence des polluants dans l'air a été approchée par voie indirecte en évaluant les taux au niveau des foliaires du site exposé à l'air libre. 


\section{Méthodes d'échantillonnage de l'eau}

Les prélèvements ont été effectués à l'aide d'une puisette en plastique reliée à une corde. La longueur de corde permet de déduire la profondeur de l'eau dans le puits étudié. Le prélèvement des eaux de puits pour l'analyse microbiologique (sulfito-réducteurs qui se développent en anaérobie), a été fait en fixant la bouteille en verre par des anneaux de cordes avec un lest afin d'atteindre le fond de puits. Lors du prélèvement, la température moyenne est restée autour de $29{ }^{\circ} \mathrm{C}$ avec un vent dominant Nord - Ouest de vitesse moyenne $3 \mathrm{~m} / \mathrm{s}$. Les échantillons ont été prélevés dans les bouteilles en plastique (analyse physico-chimique) et des bouteilles en verre (analyse microbiologique) et conservés entre 0 et $4{ }^{\circ} \mathrm{C}$. Le Tableau 1 illustre les différents points de prélèvement

\section{Méthodes d'échantillonnage des feuilles d'Azadirachta indica}

L'échantillonnage a pris en compte la position géographique des arbres, le sens du vent pendant la période d'étude (mousson), mais aussi pendant la période d'harmattan. Quatre points proches du site d'entreposage à des distances voisines (environ 50 mètres du dépôt), ont donc été retenus. Le point Fréf. situé au Nord-Ouest du dépôt hors de l'entrepôt a été retenu comme point témoin. Cette position géographique le met hors du champ des deux grands vents qui balaient le dépôt de soufre à savoir: la mousson et l'harmattan. Le Tableau 2 décrit ces différents points.

Les feuilles échantillonnées sont de même type (Azactirachta indica), sur le même type de sol. La différence entre les taux de soufre obtenus au niveau de Fréf (point témoin) et celui d'un point $\mathrm{F}$ est la variation par rapport à cette référence et notées VF.

\section{Analyse des paramètres physico-chimiques et microbiologiques \\ Analyses physico - chimiques Les foliaires}

Les paramètres analysés sont: la température; le $\mathrm{pH}$; la conductivité électrique ; les ions sulfates $\left(\mathrm{SO}_{4}{ }^{2-}\right)$; le soufre particulaire; le zinc $(\mathrm{Zn})$ et le plomb $(\mathrm{Pb})$ pour l'eau. Pour les foliaires, les paramètres retenus sont: la matière sèche, la matière organique, les sulfates; le soufre; le zinc et le plomb. Les feuilles préalablement broyées sont séchées à l'étuve à $103 \pm 2{ }^{\circ} \mathrm{C}$ jusqu'à obtention d'une masse constante selon la norme AFNOR X31 - 102 (1994).

La teneur en matière organique a été déterminée par la méthode de perte au feu. Elle est réalisée par calcination de l'échantillon à $550{ }^{\circ} \mathrm{C}$ pendant 2 heures 30 mn.

Le $\mathrm{pH}$ a été déterminé à l'aide d'un pH-mètre portable SHOT GREAT ECG 817T. La conductivité électrique est mesurée à l'aide d'un salino - conductimètre WTW 19. La méthode néphélométrique (Skoog, 2006 ; AFNOR, 1994) a été utilisée pour la détermination des sulfates suivant la norme NF T 90 - 040 de Septembre 1986 tandis que le plomb, le zinc et le soufre sont évalués par spectrométrie d'absorption atomique à l'aide d'un appareil de marque ASS Spectr AA 110 suivant la norme NF T 90 - 112 de Septembre 1986. Le dosage du soufre, du plomb et du zinc a été précédé d'un prétraitement des échantillons (minéralisation). Les échantillons d'eau sont évaporés dans des béchers de 600 $\mathrm{mL}$ à l'étuve à $105{ }^{\circ} \mathrm{C}$ pendant 5 jours; le dépôt est attaqué avec de l'eau régale $(7,5 \mathrm{~mL}$ de $\mathrm{HCl}$ concentré $36 \%+2,5 \mathrm{~mL}$ d'acide nitrique concentré $36 \%$ ) sur une plaque chauffante à feu doux, puis une élévation progressive de la température est opérée jusqu'à ébullition pendant 2 heures. On ajoute au contenu de l'acide nitrique $2 \mathrm{~N}$ dans des fioles de $100 \mathrm{~mL}$ puis on procède à la filtration (minéralisation du dépôt). Les solutions utilisées pour l'étalonnage, sont préparées à partir des standards Merck certifiés de plomb et de zinc.

\section{Analyses microbiologiques}

Pour l'analyse microbiologique, les Clostridiums sulfito réducteurs ont été déterminés dans $100 \mathrm{ml}$ et selon les méthodes d'incorporation et par filtration sur membrane. La Gélose TSN a été utilisée comme milieu de culture pour une température d'incubation de $46{ }^{\circ} \mathrm{C}$ pendant une durée de 24 heures. 


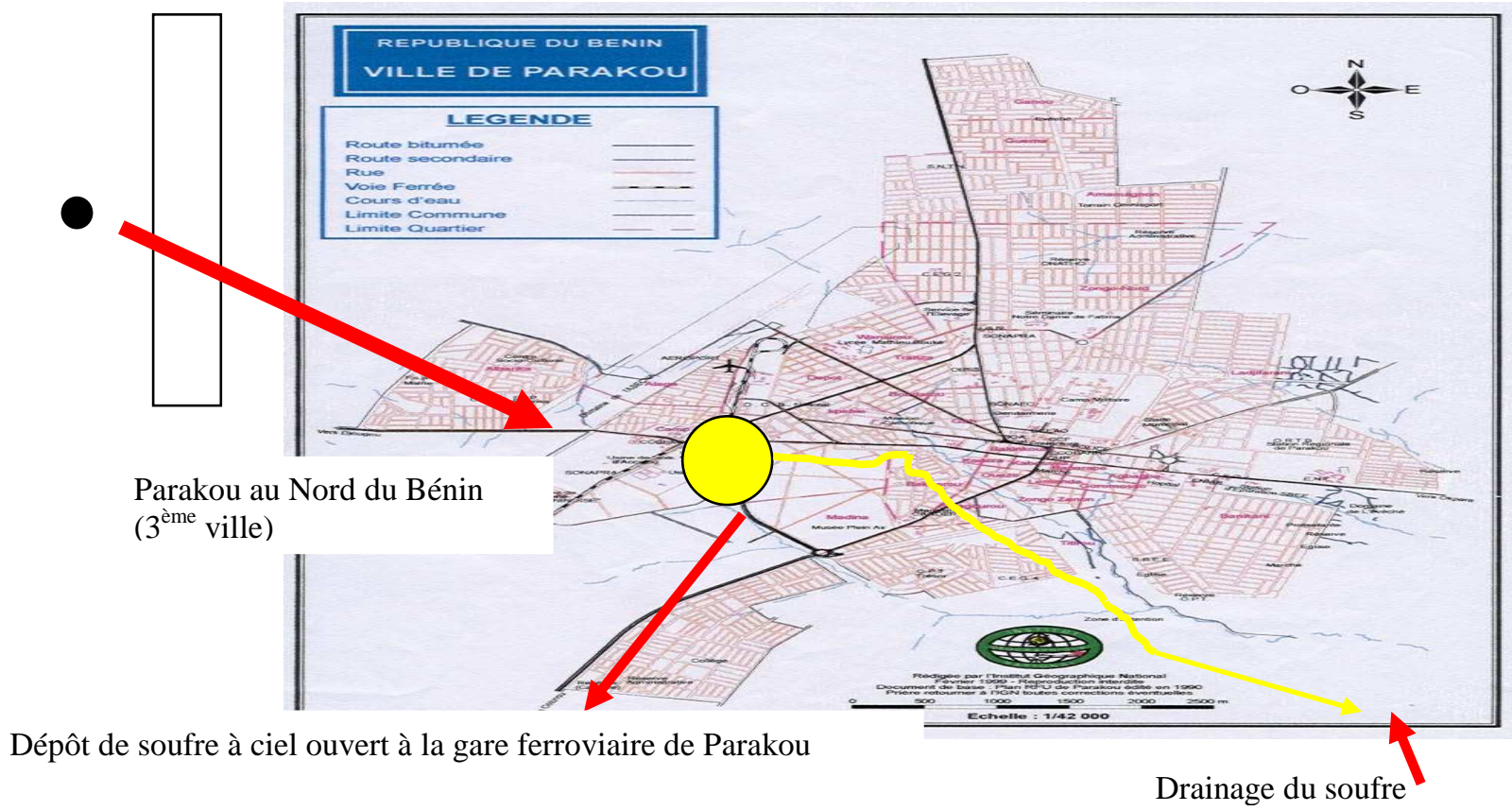

Figure 1 : Position de Parakou. 


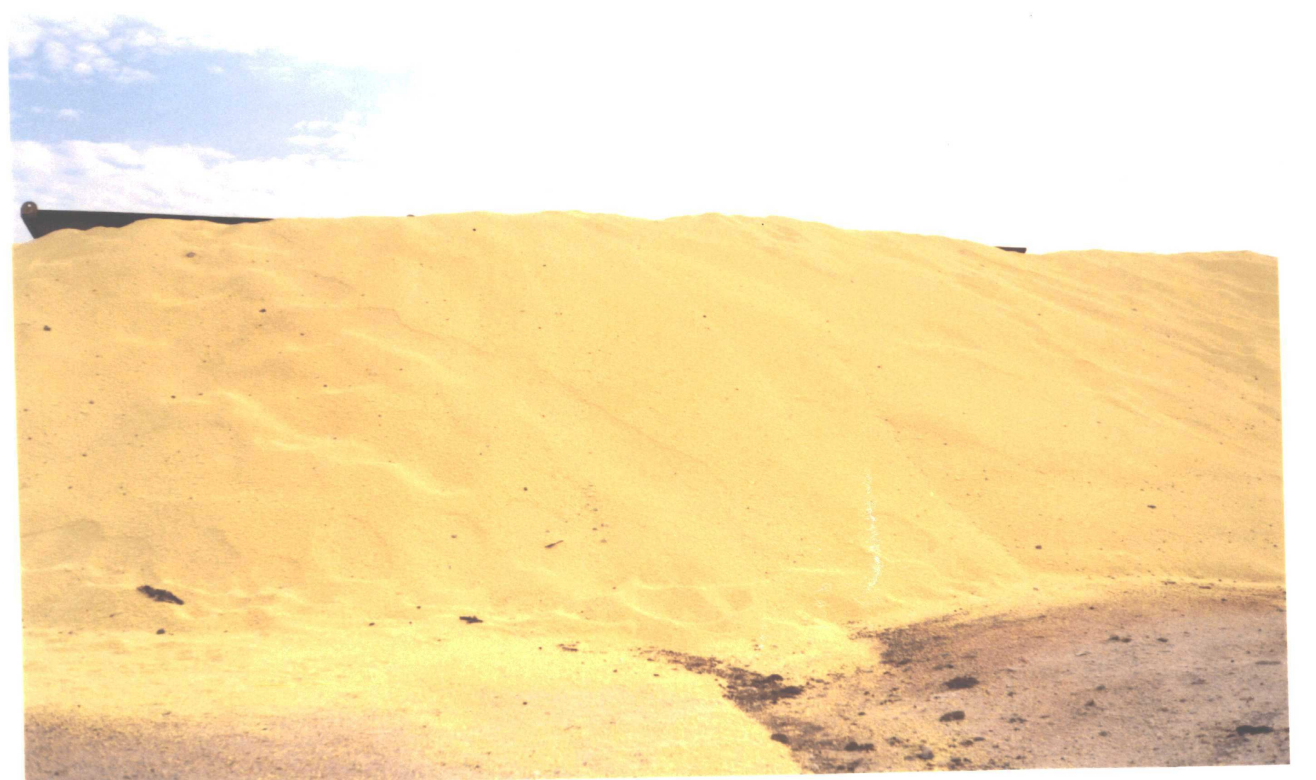

Figure 2 : Positionnement du dépôt et illustration du stockage à ciel ouvert du soufre. 
Tableau 1 : Description des points de prélèvement des échantillons d'eau.

\begin{tabular}{|c|c|c|c|c|c|}
\hline Echantillon & $\begin{array}{l}\text { Distance } \\
(\mathbf{m})\end{array}$ & Echantillon & $\begin{array}{l}\text { Distance } \\
(\mathbf{m})\end{array}$ & Echantillon & $\begin{array}{l}\text { Distance } \\
\text { (m) }\end{array}$ \\
\hline $\begin{array}{l}\text { A0 ( dépôt : } \\
\text { réf.) (puits ou } \\
\text { eau de surface) }\end{array}$ & $\mathbf{0 , 0 0}$ & A9 (puits) & 153,37 & A18 (puits) & 451,05 \\
\hline A1 (puits) & 133,99 & A10(eau de surface) & 685,29 & A19 (puits) & 983,63 \\
\hline A2 (puits) & 294,70 & A11 (eau de surface) & 1615,77 & A20 (puits) & 1160,78 \\
\hline A3 (puits) & 235,85 & A12 (eau de surface) & 2146,92 & A21 (puits) & 1588,94 \\
\hline A4 (puits) & 462,87 & A13 (puits) & 2143,53 & A22 (puits) & 1417,84 \\
\hline A5 (puits) & 942,31 & A14 (puits) & 2155,88 & $\begin{array}{l}\text { A23 } \\
\text { (eau de } \\
\text { surface ) }\end{array}$ & 1359,49 \\
\hline A6 (puits) & 1413,00 & A15 (eau de surface) & 4947,00 & $\begin{array}{l}\text { A24 (eau } \\
\text { pluviale } \\
\text { stagnant) }\end{array}$ & \\
\hline A7 (puits) & 150,33 & A16 (puits) & 2894 & $\begin{array}{l}\text { A25 (eau } \\
\text { potable de } \\
\text { robinet) }\end{array}$ & \\
\hline A8 (puits) & 150,33 & A17 (puits) & 3711,68 & & \\
\hline
\end{tabular}

Tableau 2 : Description des points de prélèvement des feuilles d'Azadirachta indica.

\begin{tabular}{ll}
\hline Echantillon & Description du point de prélèvement \\
\hline $\mathrm{F}_{1}$ à $50 \mathrm{~m}$ de l'entrepôt & Enceinte de la gare Ouest (Reférence) \\
$\mathrm{F}_{2}$ à $50 \mathrm{~m}$ de l'entrepôt & Enceinte dépôt côté Ouest \\
$\mathrm{F}_{3}$ à $50 \mathrm{~m}$ de l'entrepôt & côté Nord -Est \\
$\mathrm{F}_{4}$ à $50 \mathrm{~m}$ de l'entrepôt & Enceinte dépôt côté Sud -Ouest \\
\hline
\end{tabular}

\section{RESULTATS}

Drainage du soufre par les eaux de ruissellement

Les points ont été regroupés selon le type d'eau et le positionnement par rapport à la zone et au sens de drainage du soufre par les eaux pluviales. Les valeurs moyennes calculées et figurant dans le Tableau 6 ont permis l'obtention des courbes pour la discussion. Les puits en zone de drainage sont notés EP ZD; les puits hors zone de drainage : EP HZD ; les puits en amont du cours d'eau Boundarou: EP AZD et les eaux du cours d'eau Boundarou qui reçoit les eaux de ruissellement issues du dépôt : ESB ZD. Le Tableau 3 regroupe les valeurs moyennes des paramètres selon ces différents regroupements.

L'évolution du taux de sulfate et du $\mathrm{pH}$ est mise en relief par le Tableau 3. Cette évolution permet d'apprécier l'acidification apportée par le soufre dans les eaux de surface du site d'étude.

Toutes les températures obtenues sont supérieures à $25{ }^{\circ} \mathrm{C}$. Elles sont favorables à des activités microbiennes poussées. Les températures varient très peu pour les différents échantillons. 
Les résultats relatifs aux paramètres chimiques et microbiologiques montrent que les eaux situées en zone de drainage présentent les plus fortes teneurs en soufre $(66,45 \mathrm{mg} / \mathrm{L}$ en moyenne de sulfate pour les puits situés en zone de drainage contre des traces pour les puits hors zone de drainage). Pour les sulfito-réducteurs les eaux de surface présentent le plus fort taux. Les apports de soufre et des métaux lourds apparaissent être liés au drainage du soufre par les eaux de ruissellement comme le montre les Tableaux 5 et 6 .

Dans le cas du cours d'eau Boundarou qui reçoit les eaux de drainage, il apparaît une tendance à la diminution aussi bien des teneurs en soufre qu'en zinc et en plomb, avec une augmentation concomitante de l'acidité.

\section{Transfert du soufre dans l'air}

Les aérosols issus du dépôt, transportés par le vent se déposent sur les feuilles des végétaux situés aux alentours du site. Les éléments rencontrés dans les foliaires sont apportés par les aérosols sur ces feuilles et par le sol. La variation rapportée à la référence, permet d'estimer les taux de ces substances dans les aérosols (dépôt de poussière sur les feuilles des arbres). Les Figures 3 et 4 illustrent les résultats des analyses des feuilles d'Azadirachta indica respectivement pour le soufre et les métaux lourds.

Pour ce qui concerne les eaux, les résultats des analyses des échantillons hors zones de drainage sont regroupés dans les Tableaux 7 et 8 . Le suivi du taux de soufre, du
$\mathrm{pH}$, du zinc et du plomb pour les puits hors de la zone de drainage, montre que la proximité du dépôt n'influence pas les apports de polluant comme dans les deux cas précédents.

\section{Niveau de pollution de l'eau.}

Les résultats d'analyse des eaux de surface sont contenus dans le Tableau 9.

Les résultats obtenus montrent que les eaux de surface sont contaminées par le soufre et ses dérivés : $\mathrm{SO}_{4}{ }^{2-}$ (78,3 à 290,5 mg/L); Soufre $(568,3$ à $2086,7 \mathrm{mg} / \mathrm{L})$ et Clostridium perfergens (3000 à 40000 dans 100 ml d'eau).

Les résultats obtenus au point $\mathrm{A}_{24}$ (voir Tableau 1) qui correspond à l'eau de pluie stagnante prélevée dans l'enceinte du dépôt présentent des taux élevés de polluant: Sulfate $(9,86 \mathrm{~g} / \mathrm{L})$; Soufre $(85,6 \mathrm{~g} / \mathrm{L})$ et Clostridium Perfergens (10 000 dans $100 \mathrm{ml}$ d'eau).

\section{Les métaux dans les eaux}

Le taux de zinc est relativement élevé pour $A_{8}$ et très élevé pour $A_{24}(20,1 \mathrm{mg} / \mathrm{L}$ à l'intérieur du dépôt pour une valeur limite admissible de $5 \mathrm{mg} / \mathrm{l}$ ). Le taux de zinc a pour origine la corrosion de toiture (tôle galvanisée). Il est à remarquer que la plupart des puits ne sont pas isolés des infiltrations superficielles (absence de buse pour rendre étanche les parois).

Tableau 3: Evolution du pH et du taux de sulfate en fonction de l'éloignement par rapport au dépôt.

\begin{tabular}{lcc}
\hline $\mathbf{p H}$ & Sulfate $\mathbf{~ m g / L}$ & Distance $(\mathbf{m})$ \\
\hline 7,2 & 210,1 & 1359,49 \\
6,7 & 290,5 & 685,29 \\
7,7 & 256,6 & 1615,77 \\
7,7 & 84,5 & 2146,92 \\
7,8 & 78,3 & 4947 \\
\hline
\end{tabular}


Tableau 4: Valeur moyenne des paramètres selon les types d'eau et la zone de prélèvement.

\begin{tabular}{|c|c|c|c|c|c|c|c|c|}
\hline $\begin{array}{l}\text { Type } \\
\text { d'eau }\end{array}$ & $\mathbf{T}^{\circ} \mathbf{C}$ & pH & $\begin{array}{l}\text { Conductivité } \\
(\mathbf{m s} / \mathbf{c m})\end{array}$ & $\underset{\mathrm{mg} / \mathrm{L}}{\mathrm{SO}_{4}{ }^{2-}}$ & $\begin{array}{l}\mathrm{Pb} \\
\mathrm{mg} / \mathrm{L}\end{array}$ & $\begin{array}{l}\mathrm{Zn} \\
\mathrm{mg} / \mathrm{L}\end{array}$ & $\begin{array}{l}\text { Soufre(S) } \\
\text { mg/L }\end{array}$ & $\begin{array}{l}\text { Sulfito } \\
\text { réducteur } \\
\text { CP) }\end{array}$ \\
\hline EP ZD & $\begin{array}{l}\mathbf{2 9 , 5} \\
(28-31)^{*}\end{array}$ & $\begin{array}{l}\mathbf{6 , 4} \\
(3,8-7,3)\end{array}$ & $\begin{array}{l}\mathbf{1 , 4 4} \\
(0,5-3,43)\end{array}$ & $\begin{array}{l}\mathbf{6 6 , 4 5} \\
(0-3326,9)\end{array}$ & $\begin{array}{l}\mathbf{1 , 5 5} \\
(0-9,1)\end{array}$ & $\begin{array}{l}\mathbf{0 , 3 9} \\
(0,02-1,60)\end{array}$ & $\begin{array}{l}\mathbf{4 9 9 2 , 6 7} \\
(0-29076,5)\end{array}$ & $\begin{array}{l}\mathbf{1 3 0 1 8} \\
(30-70000)\end{array}$ \\
\hline EP HZD & $\begin{array}{l}\mathbf{2 8 , 3} \\
(27-29)\end{array}$ & $\begin{array}{l}\mathbf{6 , 6} \\
(5,3-10,1)\end{array}$ & $\begin{array}{l}\mathbf{0 , 5 4} \\
(0,34-0,84)\end{array}$ & Trace & $\begin{array}{l}\mathbf{0 , 0 1} \\
(0-0,03)\end{array}$ & $\begin{array}{l}\mathbf{0 , 0 5} \\
(0,01-0,13)\end{array}$ & $\begin{array}{l}7,09 \\
(0-24,82)\end{array}$ & $\begin{array}{l}\mathbf{8 0 4 4} \\
(50-40000)\end{array}$ \\
\hline ESB ZD & $\begin{array}{l}29 \\
(26-31)\end{array}$ & $\begin{array}{l}6,64 \\
(2,5-7,8)\end{array}$ & $\begin{array}{l}3,01 \\
(1,09-12)\end{array}$ & $\begin{array}{l}\mathbf{1 7 9 6 , 5} \\
(78,3-9859,21)\end{array}$ & $\begin{array}{l}\mathbf{0 , 1 5} \\
(0,03-0,57)\end{array}$ & $\begin{array}{l}3,38 \\
(0,02-20,1)\end{array}$ & $\begin{array}{l}\mathbf{1 5 4 0 2 , 1 2} \\
(568,3- \\
85628,68)\end{array}$ & $\begin{array}{l}14000 \\
(3000- \\
40000)\end{array}$ \\
\hline EP AZD & $\begin{array}{l}\mathbf{2 9 , 5} \\
(29-30)\end{array}$ & $\begin{array}{l}\mathbf{6 , 5} \\
(5,8-7,0)\end{array}$ & $\begin{array}{l}\mathbf{0 , 4 4} \\
(0,36-0,56)\end{array}$ & $\begin{array}{l}\mathbf{1 7 , 0 5} \\
(0-45,50)\end{array}$ & $\begin{array}{l}\mathbf{0 , 0 3} \\
-\end{array}$ & $\begin{array}{l}\mathbf{0 , 0 5} \\
(0,04-0,07)\end{array}$ & $\begin{array}{l}\mathbf{1 6 , 3 3} \\
(0-34,0)\end{array}$ & $\begin{array}{l}\mathbf{2 8 2} \\
(20-1000)\end{array}$ \\
\hline
\end{tabular}

Tableau 5 : Evolution comparée de la concentration en soufre, en fonction de l'éloignement par rapport au dépôt, dans les eaux du cours d'eau Boundarou.

\begin{tabular}{lc}
\hline Soufre mg/L & Distance (m) \\
\hline 2086,68 & 1359,49 \\
1725,7 & 685,29 \\
1463,57 & 1615,77 \\
938,62 & 2146,92 \\
568,3 & 4947 \\
\hline
\end{tabular}

Tableau 6: Evolution comparée du $\mathrm{pH}$ et des concentrations en $\mathrm{Zn}$ et $\mathrm{Pb}$, en fonction de l'éloignement par rapport au dépôt, dans les eaux du cours d'eau Boundarou.

\begin{tabular}{lccc}
\hline $\mathbf{p H}$ & Plomb (mg/L) & Zinc (mg/L) & Distance (m) \\
\hline 7,2 & 0,03 & 0,05 & 1359,49 \\
6,7 & 0,08 & 0,03 & 685,29 \\
7,7 & 0,08 & 0,03 & 1615,77 \\
7,7 & 0,06 & 0,02 & 2146,92 \\
7,8 & 0,09 & 0,03 & 4947 \\
\hline
\end{tabular}


Tableau 7: Evolution comparée du soufre dans les eaux de puits hors zone de drainage du soufre en fonction de l'éloignement par rapport au dépôt.

\begin{tabular}{lc}
\hline Soufre $(\mathbf{m g} / \mathbf{L})$ & Distance $(\mathbf{m})$ \\
\hline 0 & 150,33 \\
0 & 150,37 \\
24,8 & 1417,84 \\
568,3 & 1588,94 \\
0 & 2143,53 \\
829,9 & 2155,88 \\
\hline
\end{tabular}

Tableau 8: Evolution comparée du pH, du Zinc et du Plomb dans les eaux de puits hors zone de drainage du soufre en fonction de l'éloignement par rapport au dépôt.

\begin{tabular}{cccc}
\hline $\mathbf{p H}$ & Plomb mg/L & Zinc mg/L & Distance (m) \\
\hline 7,0 & 0,00 & 0,02 & 150,33 \\
6,6 & 0,00 & 0,02 & 150,37 \\
7,0 & 9,06 & 0,04 & 1417,84 \\
6,4 & 0,08 & 0,06 & 1588,94 \\
7,3 & 0,04 & 0,03 & 2143,53 \\
7,8 & 0,09 & 0,03 & 2155,88 \\
\hline
\end{tabular}

Tableau 9 : Résultats des analyses des eaux de surface du principal cours d'eau 'Boundarou'.

\begin{tabular}{|c|c|c|c|c|c|c|c|c|c|}
\hline Ech. & $\mathbf{T}^{\circ} \mathbf{C}$ & pH & $\begin{array}{l}\text { Cond. } \\
(\mathrm{mS} / \mathrm{cm})\end{array}$ & $\begin{array}{c}\text { Sulfate } \\
\mathrm{mg} / \mathrm{L}\end{array}$ & $\begin{array}{c}\text { Plomb } \\
\text { mg/L }\end{array}$ & $\begin{array}{c}\text { Zinc } \\
\text { mg/L }\end{array}$ & $\begin{array}{c}\text { Soufre } \\
\mathrm{mg} / \mathrm{L}\end{array}$ & $\begin{array}{c}\text { CP* } \\
/ 100 \text { ml } \\
\text { d'eau }\end{array}$ & $\begin{array}{c}\text { Distance } \\
\text { (m) }\end{array}$ \\
\hline A23 & 28 & 7,2 & 1,1 & 210,1 & 0,03 & 0,05 & 2086,68 & 40000 & 1359,49 \\
\hline A10 & 31 & 6,7 & 1,53 & 290,5 & 0,08 & 0,03 & 1725,7 & 8000 & 685,29 \\
\hline A11 & 29 & 7,7 & 1,09 & 256,6 & 0,08 & 0,03 & 1463,57 & 7000 & 1615,77 \\
\hline A12 & 28 & 7,7 & 1,12 & 84,5 & 0,06 & 0,02 & 938,62 & 16000 & 2146,92 \\
\hline A 15 & 29 & 7,8 & 1,2 & 78,3 & 0,09 & 0,03 & 568,3 & 3000 & 4947 \\
\hline Moy. & 29 & 7,42 & 1,208 & 184 & 0,068 & 0,032 & 1356,57 & 14800 & \\
\hline Min & 28 & 6,7 & 1,09 & 78,3 & 0,03 & 0,02 & 568,3 & 3000 & \\
\hline Max & 31 & 7,8 & 1,53 & 290,5 & 0,09 & 0,05 & 2086,68 & 40000 & \\
\hline Ecartype & 1,22 & 0,47 & 0,19 & 97,94 & 0,02 & 0,01 & 607,62 & 14855,98 & \\
\hline $\begin{array}{l}\text { Coef. de } \\
\text { variation (cv) }\end{array}$ & 0,04 & 0,06 & 0,16 & 0,53 & 0,29 & 0,31 & 0,45 & 1 & \\
\hline
\end{tabular}




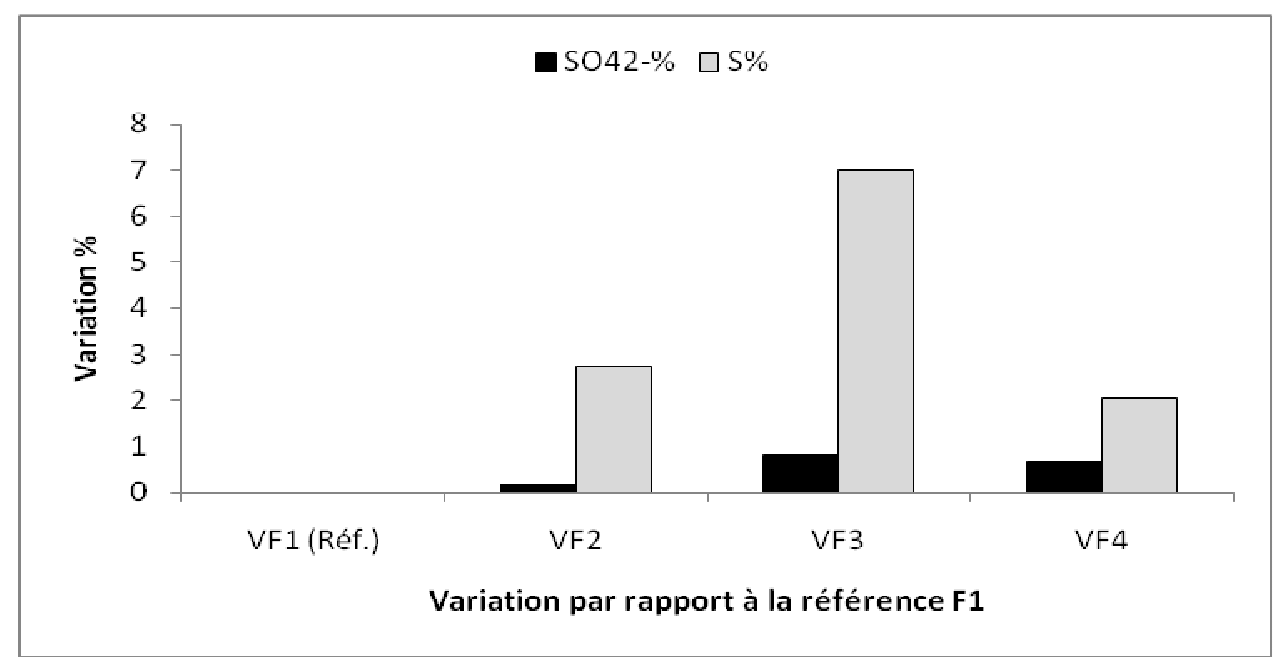

Figure 3: Taux estimé de sulfate et de soufre sur les feuilles d'Azadirachta indica.

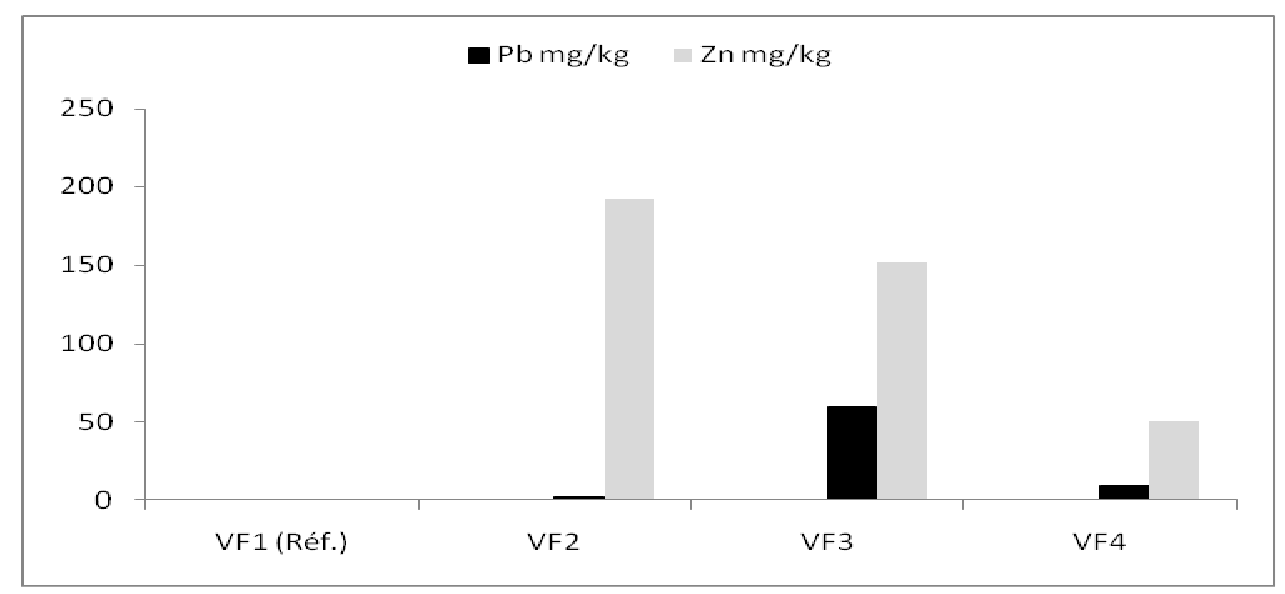

Figure 4: Taux estimé de plomb et de zinc sur des feuilles d'Azadirachta indica.

\section{DISCUSSION}

Drainage du soufre et pollution des eaux

L'analyse des résultats liés aux sulfates et au soufre, doit se faire en tenant compte de la dynamique réactionnelle chimique et biologique. A cet effet la faiblesse du taux d'un indicateur ne doit pas se comprendre comme l'absence de pollution au souffre. Ainsi, les sulfates sont utilisés par les sulfatoréducteurs anaérobies pour produire des sulfures gazeux (Lawe et al., 1993 ; Escoffier et al., 1998). Le soufre peut se présenter sous d'autres formes telles que les sulfites, les polysulfites, etc. En tenant compte des considérations précédentes et des résultats obtenus, il apparaît que hormis le point $\mathrm{A}_{25}$ correspondant à l'eau potable prélevée au robinet d'un abonné, tous les autres points de prélèvement (puits et eau de surface) présentent des valeurs supérieures aux normes OMS (250 mg/L $\mathrm{SO}_{4}{ }^{2-}$ ) pour les eaux de consommation. Pour les points $\mathrm{A}_{8}(3327 \mathrm{mg} / \mathrm{L}$ 
$\left.\mathrm{SO}_{4}{ }^{2-}\right) ; \mathrm{A}_{10}\left(291 \mathrm{mg} / \mathrm{L} \mathrm{SO}_{4}{ }^{2-}\right) ; \mathrm{A}_{11},(257 \mathrm{mg} / \mathrm{L}$ $\mathrm{SO}_{4}{ }^{2-}$ ) les concentrations en sulfate sont hors norme. Les résultats sont présentés en tenant compte du sens d'écoulement des eaux de drainage, et révèlent que le drainage du soufre par les eaux de ruissellement a contribué à la pollution des eaux par le souffre.

L'eau stagnante située à environ 50 mètres en aval du lieu de stockage, contient d'énormes quantités de soufre. A cet endroit le $\mathrm{pH}(2,5)$ mesuré est le plus faible. La hauteur d'eau est d'environ $20 \mathrm{~cm}$ et facilite son oxygénation, cependant les chlostridium perfergens, qui sont des sulfito - réducteurs de type anaérobie (Escoffier et al., 1998), s'y sont développés (1000 dans $100 \mathrm{ml}$ d'eau). Ces eaux sont issues de l'entrepôt et transportées au point $\mathrm{A}_{24}$ lors des épisodes pluvieux.

Les eaux de certains puits sont acides et hors norme OMS $(<5)$ pour l'eau potable. Il s'agit des points $A_{1}, A_{2}, A_{3}, A_{4}, A_{5}, A_{8}$ et $\mathrm{A}_{24}$. Les cas les plus extrêmes restent ceux de $\mathrm{A}_{8} \quad(\mathrm{pH}=3,8)$ et $\mathrm{A}_{24} \quad(\mathrm{pH}=2,5) . \mathrm{Ce}$ phénomène résulte de l'oxydation du soufre en $\mathrm{SO}_{2}$ qui finit par donner de l'acide $\mathrm{H}_{2} \mathrm{SO}_{3}$ puis $\mathrm{H}_{2} \mathrm{SO}_{4}$ (responsables des pluies acides). Le point $\mathrm{A}_{6}$ présente un $\mathrm{pH}$ très élevé sans doute en rapport avec la nature du sol.

Pour le plomb, le résultat obtenu montre des cas de pollution aux points $\mathrm{A}_{8}$, $\mathrm{A}_{11}, \mathrm{~A}_{12}, \mathrm{~A}_{14}, \mathrm{~A}_{15}, \mathrm{~A}_{16}, \mathrm{~A}_{20}, \mathrm{~A}_{22}$ et $\mathrm{A}_{24}$ où les valeurs obtenues sont hors normes (> 0,5 $\mathrm{mg} / \mathrm{L}$ ). Les causes peuvent être la corrosion de canalisation (sous l'effet des bactéries sulfito réductrices) mais les activités anthropiques utilisant les colorants et les carburants peuvent également en être responsables. Les forts taux de $\mathrm{Zn}$ et $\mathrm{Pb}$ dans les zones à forte concentration de soufre sont liés à l'association naturelle au soufre que manifestent ces deux métaux (Bouabdellah et al., 2008).

Les métaux lourds peuvent être entrainés vers les milieux récepteurs par lixiviation (Benmossa et al., 1994), L'influence du drainage du soufre sur les apports en métaux (Zinc et Plomb) est confirmée par les résultats. Les puits situés près du dépôt et dans la zone de drainage présentent les plus forts taux de zinc et de plomb.

Le $\mathrm{pH}$ est faible pour les eaux proches du dépôt avec une teneur également importante en soufre. La preuve de l'influence du soufre sur l'augmentation de l'acidité est établie (Heslop et Robinson, 1973). Les tableaux permettent un suivi en fonction aussi bien de l'éloignement que de la situation ou non des points dans la zone de drainage du soufre par les eaux de ruissellement.

Cette tendance d'évolution est pratiquement la même pour les eaux de surface (Boudarou) hormis pour le cas du plomb. Mais les investigations menées sur le terrain ont révélé que le point correspondant à la distance $1478 \mathrm{~m}$ du dépôt avait servi auparavant de dépotoir sauvage de déchets ménagers. On peut donc attribuer le taux élevé de plomb au niveau de ce point singulier à une pollution liée à cette activité antérieure (Bouabdellah et al., 2008).

\section{Transfert du soufre dans l'air et pollution des aérosols}

Les résultats d'analyse du soufre et de ses dérivés dans quatre échantillons de foliaire de même type, situés à égale distance du site dans des directions différentes le confirment. En effet, le taux (en pourcentage massique) de soufre dans les aérosols varie de $0,27 \%$ à 6,27 $\%$. Les valeurs les plus élevées sont obtenues pour les arbres situés dans le sens du vent dominant (F3 et $\mathrm{F} 4)$ bien qu'ils soient à équidistance du dépôt avec $\mathrm{F} 1$ et $\mathrm{F} 2$. Les résultats obtenus coïncident avec les facteurs de proximité et du sens dominant de la direction du vent. La présence du soufre dans les aérosols voire dans l'air ambiant est établie. Le transport du soufre et de ses dérivés est assuré par le vent vers des sites hors de l'entrepôt. Les points situés au Sud Ouest de l'entrepôt reçoivent une forte teneur de soufre pendant l'harmattan. Les apports du soufre sont liés au régime du vent autour du dépôt. 


\section{Conclusion}

Les résultats que nous avons obtenus montrent que la présence du dépôt de soufre stocké en plein air et soumis aux intempéries (pluies et vents) a occasionné la pollution des eaux de surface et souterraines. Cette pollution au soufre a été amplifiée par sa migration dans le sol et l'air. Les transformations chimiques $\mathrm{du}$ soufre ont entraîné l'acidification des eaux de pluie, du sol et des eaux autour du site de dépotage. Associées à ce dépôt, on rencontre des bactéries sulfato-réductrices (Clostridium perfergens). Les acides dérivés du soufre et les métaux toxiques issus de la corrosion des matériaux autour du site, ont eu pour conséquence la dégradation de la qualité des eaux des puits par la modification des caractéristiques physico-chimiques et microbiologiques avec :

- un taux moyen variant de 282 Clostridium perfergens / $100 \mathrm{~mL}$ d'eau (puits hors de la zone de drainage) et 13018 Clostridium perfergens $/ 100 \mathrm{ml}$ (puits situés en zone de drainage du soufre) ;

- des concentrations de $0,01 \mathrm{mg} \mathrm{Pb} / \mathrm{L}$ et $0,05 \mathrm{mg} \mathrm{Zn} / \mathrm{L}$ (puits hors site de stockage) à $1,55 \mathrm{mg} \mathrm{Pb} / \mathrm{L}$ et $0,39 \mathrm{mg} / \mathrm{l} \mathrm{Zn}$ (puits en zone de drainage).

Cette pollution a également contribué à la dégradation du sol par acidification et accroissement des taux de soufre et de métaux toxiques de même qu'à celle de l'air par une augmentation des taux de soufre et dérivés des aérosols. La contamination de ces milieux récepteurs a eu des implications sanitaires voir socio-économiques sur les populations situées aux alentours du site. Le dépôt de soufre apparaît comme principal facteur de dégradation des eaux du cours d'eau Boundarou et des puits à Parakou.

\section{BIBLIOGRAPHIE}

AFNOR. 1994. Qualité des Eaux, Environnement. AFNOR Edition: Paris.

Benmoussa H, Tyagi DR, Campbell PGC. 1994. Biolixiviation des métaux lourds et stabilisation des boues municipales : effet de la forme du soufre élémentaire utilisé comme substrat. Rev. Sci. Eau, 7(3): 235250.

Bouabdellah M, Boudchiche L, Ouahabi B, Naciri T. 2008. Le soufre associé aux minéraux $\mathrm{Pb}-\mathrm{Zn}$ de type Mississipi Valley de la chaîne des Beni Snassen orientaux (Maroc nord oriental). Compte Rendu Geosciences, 340(12): 822-828.

Braun - Fahrlander C, Ackermann - Liebrich U, Schwartz J, Gnehm HP, Rutishauser M, Wanner HU. 1992. Air pollution and respiratory symptôme symptoms in preschool children. Am. Rev. Respir. Dis., 145: $42-47$.

Chailleux E, Guyon C, Tadeï F, Bouillard J, Pioche D. 1990. Asthme et pollution atmosphérique. Une étude des admissions à l'hopital de Saint - Nazaire. Rev. Mal. Resp., 7: 563-568.

Dab W, Medina S, Quenel P, Le Moulec Y, Le Tertre A, Thelot B, Montel C, Eaton, AD. 1995. Quality Assurance (QA) / Quality Control (QC) for Organique Compounds in Standard Method. (19ème Ed). Washington.

Escoffier S, Olivier B, Le Mer J, Garcin J, Roger P. 1998. Evidence and quantification of thiosulfate reducers unable to reduce sulfate in ricefield soils. Eur. J. Soil Biol., 34(2): 69-74.

Freney JR, Jacq VA, Baldensperger JF. 1982. The significance of biological sulphur cycle in rice production. In: Microbiology of Tropical Soiland Plant Productivity, Dommergue YD, Diem HG (Eds). Martinus Nijhoff/w Junk Pub: The Hague; 271 - 317 .

Lameloise P, Pirard P, Momas I, Ferry R, Festy B. 1996. Short term respiratory health effects of ambient air pollution results of the APHEA project in Paris. $J$. Epidemiol. Cimm. Health, 50(1): 42-46.

Heslop RB, Robinson PL. 1973. Chimie Inorganique (2 ème édn). Flamarion: Paris.

Lawe SE, Jain MK, Zeikus JG. 1993. Biology, ecology, and biotechnological applications of anaerobic bacteria adapted to environmental stress in temperature, 
$\mathrm{pH}$, salinity, or substrates. Microbiol. Rev., 57: 451 - 509 .

Le CEDRE: Centre de documentation, de Recherche et d'Expérimentation sur les pollutions accidentelles des eaux ( en ligne). CEDRE, 2010 (réf. Du 29/11/2010. Disponible sur World Wide Web < http: // www.cedre/fr/accident/ detroit_kertch/soufre.php>

Le Roux P, Boileau C, Loisel I, Roger AM, Boulloche J, Czernichow P, Le Luyer B. 1997. Asthme chez l'enfant et pollution atmosphérique, étude épidémiologique dans l'environnement du Havre. Rev. Fr. Allergol., 37(2): 176-182.

Mama D, Garba Z, Agossou SS, Ouinkoun G. 2001. Evaluation de l'impact environnemental du dépôt de soufre à la gare OCBN de Parakou. Rapport d'étude de l'Agence Béninoise pour l'Environnement, (Ed) ABE Cotonou .

Marzin C, Lemoullec Y, Ancelle Th, Juhel J, Festy B, Pretet S. 1993. Asthme, pollution atmosphérique urbaine et météorologie. Rev.Mal. Resp., 10: 229_ 235.
Sigg L, Stumm W, Behra P. 1994. Chimie des Milieux Aquatiques. Chimie des Eaux Naturelles et des Interfaces dans l'Environnement (2 ${ }^{\text {ème }}$ édn). Masson: Paris, Milan-Barcelone .

Skoog West Hôller. 2006. Chimie Analytique ( $7^{\text {ème }}$ édn). Traduction de la version américaine, Presse de Beeck: Paris.

SERHAU. 1999. Actualisation du plan directeur d'urbanisme: analyse urbaine de la ville de Parakou. Rapport d'étude technique, (Ed) SERHAU SA, Cotonou.

Soyseth V, Kengerrud J, Haarr D, Straud O, Boile R, Boe J. 1995. Relation of exposition to airway irritants in infancy to prevalence of bronchial hyper responsiveness in schoolchildren. Lancet, 345: 217 - 220 .

Wind T, Conrad R. 1995. Sulphur compounds, potential turnover of sulphate reducing bacteria in planted and unplanted paddy soils. FEMS Microbiol. Ecol., 18: 257-266. 